\title{
SUIVI DES PÊCHES DE GÉNITEURS D'OMBLE CHEVALIER (SALVELINUS ALPINUS L.) SUR LA PARTIE FRANÇAISE DU LAC LÉMAN DE 1982 A 1987. PREMIĖRES DONNÉES SUR LE PACAGE LACUSTRE DE L'OMBLE.
}

\author{
A. ChAMpigneulle (1), M. MiChoud (2), D. GerdeAuX (1), C. Gillet (1), \\ J. GUILLARD (1) et R. ROJAS-BELTAN (1)
}

(1) Station d'Hydrobiologie Lacustre, INRA, BP $11 \mathrm{~F}$, 75, Avenue de Corzent, 74203 THONON, France.

(2) D.D.A. de Haute-Savoie, Pisciculture Domaniale de Rives, quai de Rives, 74203 THONON, France.

Reçu le 25 avril 1988

Accepté le 11 juillet 1988

Received 25 April, 1988

Accepted 11 July, 1988

\section{RÉSUME}

L'article présente les caractéristiques des pêches de reproducteurs d'omble chevalier (Salvelinus alpinus) pratiquées sur des omblières profondes de la partie française du Léman de 1982 à 1987. Une partie des plus petits alevins nourris $(3-5 \mathrm{~cm})$ déversés au Léman au cours de l'été 1983 a été marquée. Le suivi des recaptures au moment des pêches de reproducteurs 'de 1985 à 1987 indique que $50 \%$ au moins des géniteurs (cohorte 1983) capturés proviennent du repeuplement. La valeur de l'omble pour le pacage lacustre est discutée.

\section{SURVEY OF THE CATCHES OF ARTIC CHARR (SALVELINUS ALPINUS L.) SPAWNERS ON THE FRENCH SHORE OF LAKE LEMAN FROM 1982 TO 1987. FIRST DATA ON THE EFFICIENCY OF LAKE-RANCHING WITH CHARR.}

\section{SUMMARY}

The paper presents the main characteristics of the catches of artic charr (Salvelinus alpinus) spawners on the deep spawning grounds of the French shore of Lake Leman from 1982 to 1987 . A part of the smallest $(3-5 \mathrm{~cm})$ prefed fry stocked in Lake Leman during the summer 1983 has been marked. The survey of catches of spawners from 1985 to 1987 indicated that at least $50 \%$ of catches (cohort 1983) are stocked fish. The value of charr for lake-ranching is discussed.

\section{INTRODUCTION}

L'omble chevalier (Salvelinus alpinus L.) est une espèce indigène dans le Léman (fig. 1), lac de 58.240 hectares, partagé entre la Suisse et la France (LAURENT, 1972). L'espèce est capturée à l'aide de filets maillants par les pêcheurs professionnels et à la traîne par les pêcheurs amateurs en bateau. La pêche est ouverte de la mi-janvier à la mi-octobre et la taille minimale de capture est de $27 \mathrm{~cm}$ (longueur totale). Trois grandes périodes peuvent être distinguées dans les captures totales (France et Suisse) d'ombles par pêche professionnelle depuis 1950 (fig. 2a). De 1950 à 1963 les captures sont restées à un niveau élevé compris entre 10 et $31 \mathrm{t} / \mathrm{an}$. Après une chute très marquée lors des années 60, elles sont restées à un niveau bas, compris entre 2 et $6 \mathrm{t} / \mathrm{an}$, de 1967 à 1981 (fig. 2), alors que le Léman devenait mésoeutrophe (CIPEL, 1984). Les prises ont ensuite augmenté variant entre 6 et 9,5 t/an au cours de la période 1982-87. Les captures par la pêche amateur française, évaluées depuis 1982 ont varié entre 1,5 et 3,5 t/an : celles de la pêche amateur suisse évaluées depuis 2 ans sont voisines de 5 tonnes/an. En 1986, 15 tonnes d'ombles ont été déclarées et la figure $2 b$ précise la répartition des captures en fonction des diverses catégories de pêcheurs. L'omble chevalier sauvage fait l'objet d'une forte demande et a une très bonne valeur marchande puisqu'il est vendu à 65-70 F le kg par les pêcheurs professionnels.

La connaissance précise de certaines omblières profondes (50-100 $\mathrm{m}$ ) a été exploitée depuis près de 100 ans (CRETTIEZ, 1906 ; DUSSART, 1952) pour la capture aux filets de géniteurs au cours de la période de reproduction, sous contrôle de l'administration. Sur ces omblières, la fraie se déroule principalement entre le début décembre et la mi-janvier. Jusqu'à une époque récente, les alevins issus des œufs ainsi récoltés étaient déversés (stades alevins vésiculés ou nourris) sans que l'on cherche à connaître leur devenir. 


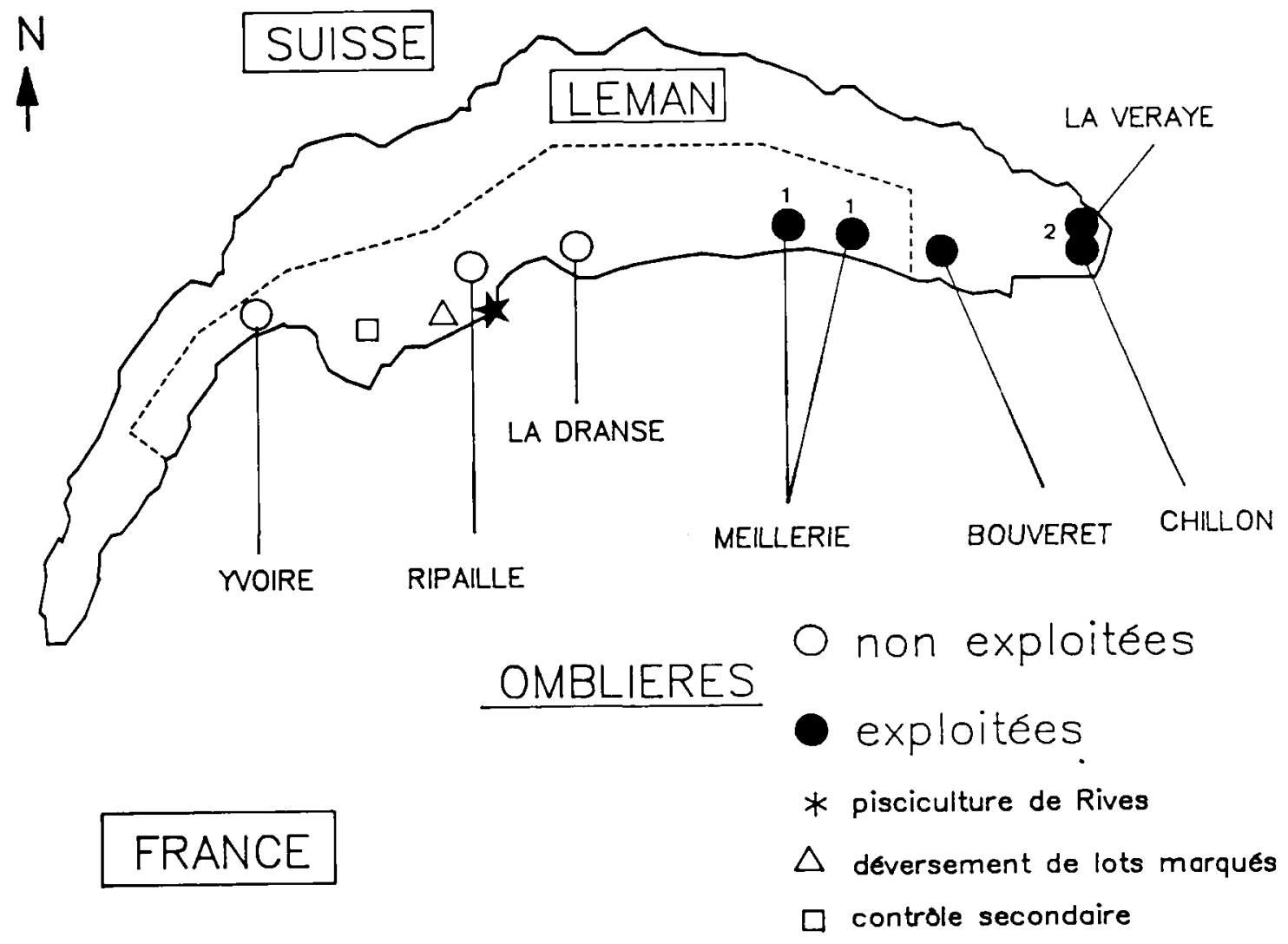

Figure 1 : Le lac Léman (---) frontière franco-suisse sur le le lac. Omblières connues: $(\bullet)$ pêchées : $(O)$ non péchées. Lot marqué en 1983 : site d'élevage $(\star)$; site de déversement $(\Delta)$; sites de contróle des captures : site princlpal, décembre 1985-86-87 (1); (2) site complémentaire, décembre 86-87; site secondaire, août 1985 (ㅁ).

Figure 1 : The lake Leman (---) boundary between France and Switzerland, on the lake. Known spawning grounds : $(\bullet)$ explolted ; $(0)$ unexploited. Survey of the marked group released in 1983 : rearing place $(\star)$; restocking place $(\Delta)$; place of control of recaptures ; main place, december 1985-86-87 (1) ; (2) complementary place, december 1986-87; secondary place, august 1985 ( $\square$ ).

Les piscicultures de repeuplement se sont cependant progressivement orientées vers la production d'estivaux pour les raisons suivantes:

- faible efficacité des lâchers d'alevins vésiculés dans le cas des grands lacs (CHAMPIGNEULLE, 1985)

- possibilité d'une production de masse à faible coüt ( $\leqslant 1$ F.F. pièce, MICHOUD, comm. person.) dans les conditions locales.

- facilité d'élevage et bonne croissance des juvéniles dans la gamme de température $\left(6-13^{\circ} \mathrm{C}\right)$ d'eau disponible par pompage au lac entre le printemps et l'été.

Depuis 1982, la station INRA de Thonon a développé, en relation avec la DDA de haute-Savoie, un programme de recherche-développement visant à connaître et à améliorer l'efficacité des opérations de pêche de reproducteurs et de repeuplement en omble chevalier. Ce programme comporte les trois aspects complémentaires suivants :

- Suivi des pêches de reproducteurs avec deux objectifs: la caractérisation des géniteurs capturés et l'augmentation du rendement des pêches vis-à-vis du nombre d'œufs récoltés.

- La mise au point d'une technique de marquage (CHAMPIGNEULLE et ESCOMEL, 1984) d'omble chevalier de petite taille (à partir de $3 \mathrm{~cm}$ ), puis son utilisation lors d'une première campagne en 1983 de marquage-suivi des recaptures afin de tenter d'évaluer le devenir des estivaux relàchés.

- La poursuite, à la pisciculture expérimentale de I'INRA à Thonon, des premiers travaux (LAURENT, 1982a) sur l'élevage de l'omble chevalier par la maîtrise de la reproduction et de la qualité des pontes (Institut de Limnologie, 1986; GILLET, données non publiées). 
POIDS ( Kg)

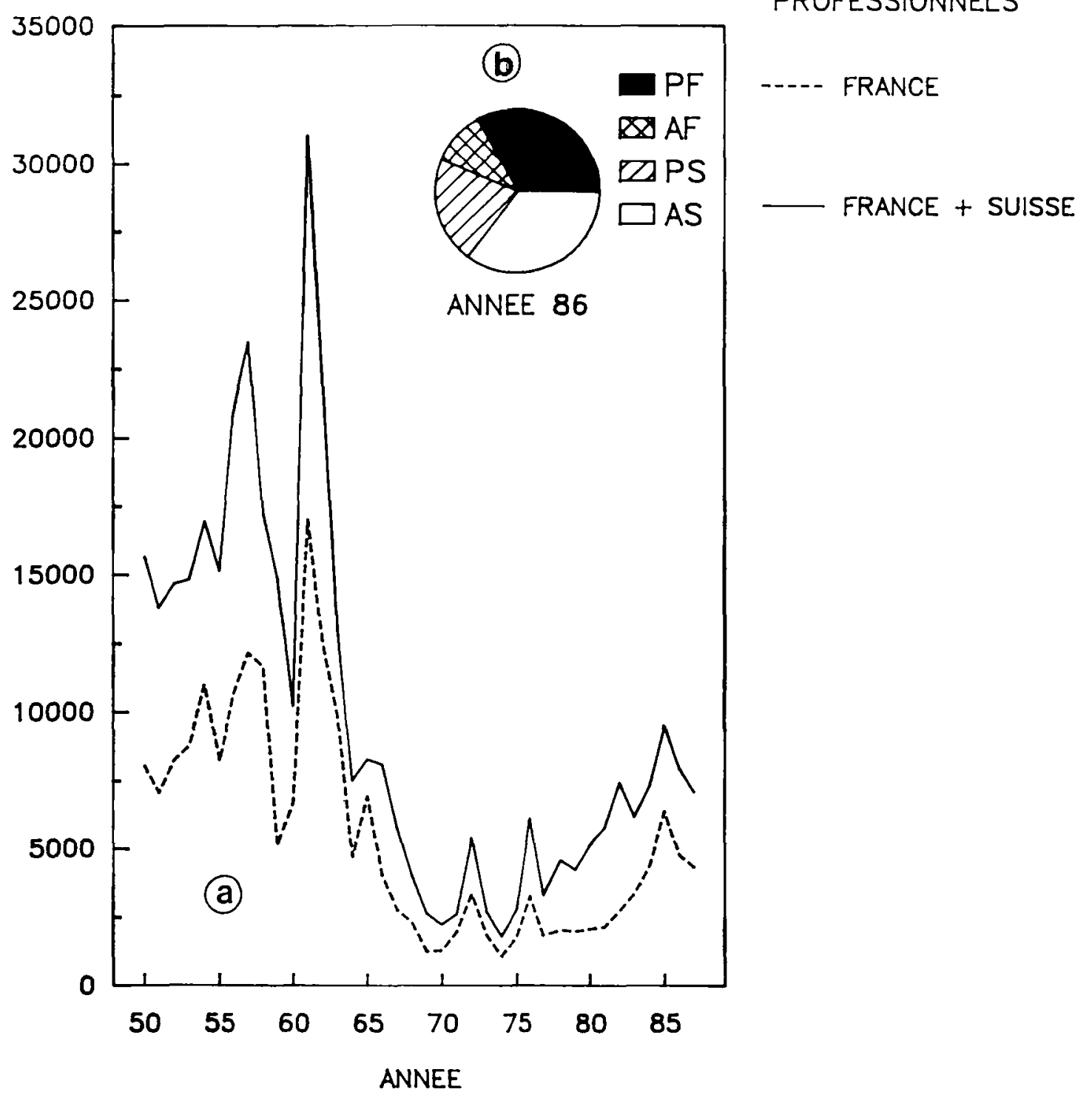

PROFESSIONNELS

Figure 2 : Statistiques annuelles de pêche en omble chevaller au Léman (données DDA en France et conservation de la Faune en Suisse).

2a - Captures déclarées par les pêcheurs professionnels depuis $1950:(\longrightarrow)$ France et Suisse ; (---) France.

2b - Repartition des captures totales (15 t) en 1986 : professionnels : (PF) France ; (PS) Suisse; amateurs : (AF) France ; (AS) Suisse.

Figure 2 : Data of annual catches of charr in Leman lake. (Origin of data : DDA in France and Animal life Preservation Service in Switzerland).

$2 a-$ Catches by professionnal tishermen, period 1950-87:(——) France and Switzerland ; (----) France.

$2 \mathrm{~b}$ - Distribution of total catches (15 t) in 1986 ; professionnal fishery : (PF) France ; (PS) Switzerland ; recreationnal fishery : (AF) France ; (AS) Switzerland.

Le présent article porte sur les deux premiers points du programme. Notons enfin que le Service de la Conservation de la Faune en Suisse mène d'importants travaux complémentaires, d'une part sur le suivi en bathyscaphe des omblières naturelles et d'autre part sur la réalisation, en 1984 et 1985, de deux nouvelles campagnes de marquage d'estivaux d'omble dont les recaptures sont suivies sur l'ensemble du Léman. 


\section{MATÉRIEL ET MÉTHODES}

\subsection{Suivi des pêches de reproducteurs}

Sur la partie française du Léman, des œufs d'ombles sont occasionnellement récoltés lors des pêches de reproducteurs de corégones (Coregonus sp.) au cours de la deuxième quinzaine de décembre et au moment de la réouverture de la pêche des Salmonidés à la mi-janvier. Cependant l'essentiel de la récolte actuelle d'œufs d'ombles provient de pêches spécifiques réalisées en décembre par des pêcheurs professionnels, sous contrôle de la DDA avec des filets maillants appelés "petits pics" (80-100 m de long, 6-8 m de haut "mailles ouvertes") posés par 80 à $120 \mathrm{~m}$ de fond sur les omblières de Meillerie-Locum (dites de Meillerie) (fig. 1). Ce sont ces pêches spécifiques qui ont été suivies, six années consécutives, de 1982 à 1987 . Au cours de cette période, les filets ont été tendus en milieu d'après-midi $(15 \mathrm{~h})$ et relevès le lendemain matin $(8 \mathrm{~h})$. L'unité d'effort de pêche choisie, appelée "pose", correspond à la tendue d'un filet pendant une nuit. L'effort de pêche total a varié entre 17 et 35 poses selon l'année. A chaque relevé, toutes les captures ont été dénombrées et classées selon trois catégories: mâles murs ( $\delta \mathrm{M})$, fermelles ovulées dites femelles mures ( $Q M$ ) et femelles matures mais non ovulées dites femelles non müres ( $\$ N M$ ). Le poids total global des ombles capturés à chaque pêche a été relevé. De 1982 à 1987, la taille (longueur totale - LT) a été mesurée sur un échantillon variant selon l'année entre 344 et 1992 individus et représentant de 30 à $100 \%$ des captures totales. La fécondation a été réalisée sur le site pour les ovules des femelles mures et les œufs ont été mis après dénombrement en incubation à la pisciculture de la DDA à Thonon.

Deux pratiques de pêche de reproducteurs ont été testées. De décembre 1982 à 1984 , les filets utilisés avaient un maillage de $40-45 \mathrm{~mm}$ (côté de maille). La majorité des femelles capturées non mures au moment de la pêche n'a pas été conservée en vie et n'a pas fourni d'œufs. Cependant, des essais limités ont permis de mettre en évidence la possibilité de recueillir des œufs supplémentaires (non pris en compte dans le rendement en œuf́s des pêches de 1982 à 1984) en stabulant les femelles non mures et encore vivantes (CHAMPIGNEULLE et al., 1983). Par ailleurs, pendant les pêches de 1983 et 84 , des essais limités ont été pratiqués avec des filets ayant un maillage plus élevé (48 à $50 \mathrm{~mm}$ ) afin de comparer les caractéristiques des captures (sexe-ratio, taille).

Les résultats de ces divers essais ont conduit à modifier le déroulement des pêches de 1985-86 et 87 en vue d'augmenter le rendement en œufs (nombre d'œufs récoltés par kg d'omble capturé ou par femelle capturée). L'utilisation de filets à $48-50 \mathrm{~mm}$ de côté de maille a été généralisée. Toutes le femelles non mûres encore vivantes ont été stabulées en pisciculture (température: $5-8^{\circ} \mathrm{C}$ ) et régulièrement contrôlées (chaque 2-3 jours). Les ovules ont été prélevées sur les femelles ovulant spontanément.

\subsection{Campagne (1983) de marquage et sulvi des recaptures}

Le tableau I précise les caractéristiques des relâchers de juvéniles d'omble chevalier au Léman en 1983. Les alevins $(3-10 \mathrm{~cm})$ ont été produits en pisciculture, nourris avec des granulés à truites. Ils ont été dispersés du début juillet au début septembre sur la rive française (244.400 estivaux) et la rive suisse (124.700 estivaux) du Léman. Les relâchers ont été réalisés au-delà de la beine, sur le talus du mont, afin d'éviter la prédation par les perches. Les juvéniles plongent très rapidement après leur relâcher. Un lot de 26.500 alevins nourris ( $[=4 \mathrm{~cm} ; \vec{P}=0,5 \mathrm{~g}$ ) issus de la production française a été marqué par cautérisation de la nageoire adipeuse selon la méthode testée et décrite par CHAMPIGNEULLE et ESCOMEL (1984). Ils ont été relâchés du 11 au 27 juillet 1983 en bordure du mont face à la Station INRA, à $25 \mathrm{~km}$ des omblières de Meillerie (fig. 1). Le lot d'alevins nourris marqués représente $10.8 \%$ des relâchers sur la rive française et $7,2 \%$ du nombre total d'alevins nourris relâchés au Léman en 1983. Environ $60 \%$ du nombre total d'alevins prégrossis relâchés se situaient dans la même gamme de taille que celle du lot marqué, alors qu'environ $40 \%$ avaient une taille supérieure comprise entre 6 et $10 \mathrm{~cm}$.

La présence d'ombles marqués a été essentiellement recherchée dans des échantillons de géniteurs capturés sur les principales omblières exploitées, celles de Meillerie lors des pêches de reproducteurs en décembre 1984-85-86 et 87. Les ombles marqués recapturés ont été mesurés (longueur totale), sexés et des écailles ont été prélevées afin d'avoir des écailles de référence d'omble d'âge connu. En décembre 1986, des écailles ont éṫ́ prélevées sur un échantillon de 266 mâles et 66 femelles capturés sur les omblières de Meillerie afin d'établir une grille de référence taille-âge, par sexe. Un suivi complémentaire a également été réalisé (RUBIN, comm. person.) lors des pêches de reproducteurs pratiquées en décembre 1986-87 avec des filets (maille $50 \mathrm{~mm}$ ) sur les omblières les plus éloignées (Chillon, Suisse) du site de déversement du lot marqué, soit à $15 \mathrm{~km}$ à l'est de celles de Meillerie, et à $40 \mathrm{~km}$ de la zone de déversement du lot marqué. Notons enfin qu'un contrôle ponctuel a été pratiqué au cours de la saison de pêche dans un échantillon de 338 ombles pêchés au filet maillant de la mi-aoüt à la fin septembre 1985 en baie d'Excenevex, à $5 \mathrm{~km}$ à l'est du site de déversement des ombles marqués (fig. 1). 
Tableaul : Bilan des repeuplements en juvéniles d'omble chevaller dans le Léman en 1983. Les valeurs entre parenthèses indiquent le pourcentage d'alevins nourris marqués.

Table 1 : Data on the restocking of Leman Lake with young artic charrs in 1983. The values in brakets indicate the percentage of marked fed-fish.

\begin{tabular}{|c|c|c|c|c|c|}
\hline & $\left|\begin{array}{l}\text { Alevins } \\
\text { vesiculess }\end{array}\right|$ & \multicolumn{4}{|c|}{ Alevins nourris } \\
\hline & Nonbre & Noubre & Date & Taille & lieu \\
\hline 0. & $\cdots$ & $\cdots$ & $---1+--4$ & . & \\
\hline SUISSE & & & & & \\
\hline Canton de Genève & 43.800 & 10.000 & fin juin & $3-4$ & Rive Genève \\
\hline Canton de Vaud & 0 & 104.700 & $\begin{array}{l}\text { juillet à } \\
\text { début septembre }\end{array}$ & $4-10$ & Rive Vaud \\
\hline Canton du Valais & 0 & 10.000 & début septembre & $7-10$ & Rive Valais \\
\hline Total Suisse & 43.800 & 124.700 & & $3-10$ & $\begin{array}{l}\text { Rive Suisse } \\
\text { dont } 50.000 \\
\text { Bouveret d } \\
\text { Montreux }\end{array}$ \\
\hline FRANCE & & & & & \\
\hline non marqués & 64.000 & 217.900 & juillet-zoût & $3-8$ & Rive française \\
\hline $\begin{array}{l}\text { marqués } \\
(\% \text { du total })\end{array}$ & 0 & $\begin{array}{c}26.500 \\
(10,86 \%)\end{array}$ & juillet & $\begin{array}{c}3-5 \\
(\bar{L} T=38,9 \pm 0,6 \mathrm{~mm}) \\
\overline{\mathrm{P}}=554.9\end{array}$ & Thonen \\
\hline Total & 64.000 & 244.400 & & $3-8$ & Rive française \\
\hline FRANCE + SUISSE & & & & & \\
\hline non marqué & 107.800 & 342.600 & & $3-10$ & $\begin{array}{l}\text { Ensemble rives } \\
\text { Léman }\end{array}$ \\
\hline $\begin{array}{l}\text { marqués } \\
(\because \text { du total) }\end{array}$ & 0 & $\begin{array}{l}26.500 \\
(7,18 \%)\end{array}$ & & $3-5$ & Thonon \\
\hline Total & 107.800 & 369.100 & & $3-10$ & \\
\hline
\end{tabular}

\section{RÉSULTATS}

\subsection{Bilan général des pẻches (Tab II)}

\subsubsection{Captures}

De 1982 à 1987, les captures totales annuelles réalisées en décembre sur les omblières de Meillerie ont varié entre 426 et 2.164 géniteurs d'ombles pour 155 à $916 \mathrm{~kg}$. Il y a eu une très nette augmentation des captures par unité d'effort à partir de 1983 ( $T$ ab. II). En effet, les captures par pose de filet de $40 \mathrm{~mm}$ sont passées de 25 ombles/pose ( $9 \mathrm{~kg} /$ pose) en 1982 à $91-116$ ombles/pose (32 à $44 \mathrm{~kg} / \mathrm{pose}$ ) respectivement en 1983 et 1984 . Avec les filets de $50 \mathrm{~mm}$, les captures par pose ont été très fortes en 1985 (105 ombles/pose pour $45 \mathrm{~kg} / \mathrm{pose})$. Elles ont ensuite baissé, tout en restant élevées avec 62 ombles/pose pour $26-30 \mathrm{~kg} /$ pose (Tab. II). Quels que soient l'année et le mode de pêche, les captures (Tab. II) sont essentiellement constituées par des mâles (70 à $88 \%$ ) qui sont spermiants pour la plupart. Par contre, it y a chez les femelles capturées un pourcentage important, 29 à $45 \%$ selon l'année, de femelles "non müres". Sur l'ensemble des captures réalisées de 1985 à 
1987 le pourcentage de femelles est significativement $(p<0,001)$ plus élevé $(25 \%)$ que celui $(14 \%)$ observé dans les captures de 1982 à 1984. Pour cette dernière période le poids moyen des ombles capturés variait selon l'année entre 354 et $380 \mathrm{~g}$; il était plus élevé ( 423 à 487 selon l'année) au cours de la seconde période.

Tableaull : Bilan des pêches de reproducteurs d'omble sur les omblières de Melllerie, en décembre de 1982 à 1987 et selon deux modes de péche. (N) : nombre; (P): poids ; (M) : mûres ; (NM) : non múres.

Tablell : Data on the catches of charr spawners on the spawning grounds of Meillerie, in december 1982 to 1987 according to two fishing technics. (N) : number ; $(P)$ : weight ; (M) : ripe ; (NM) : unripe.

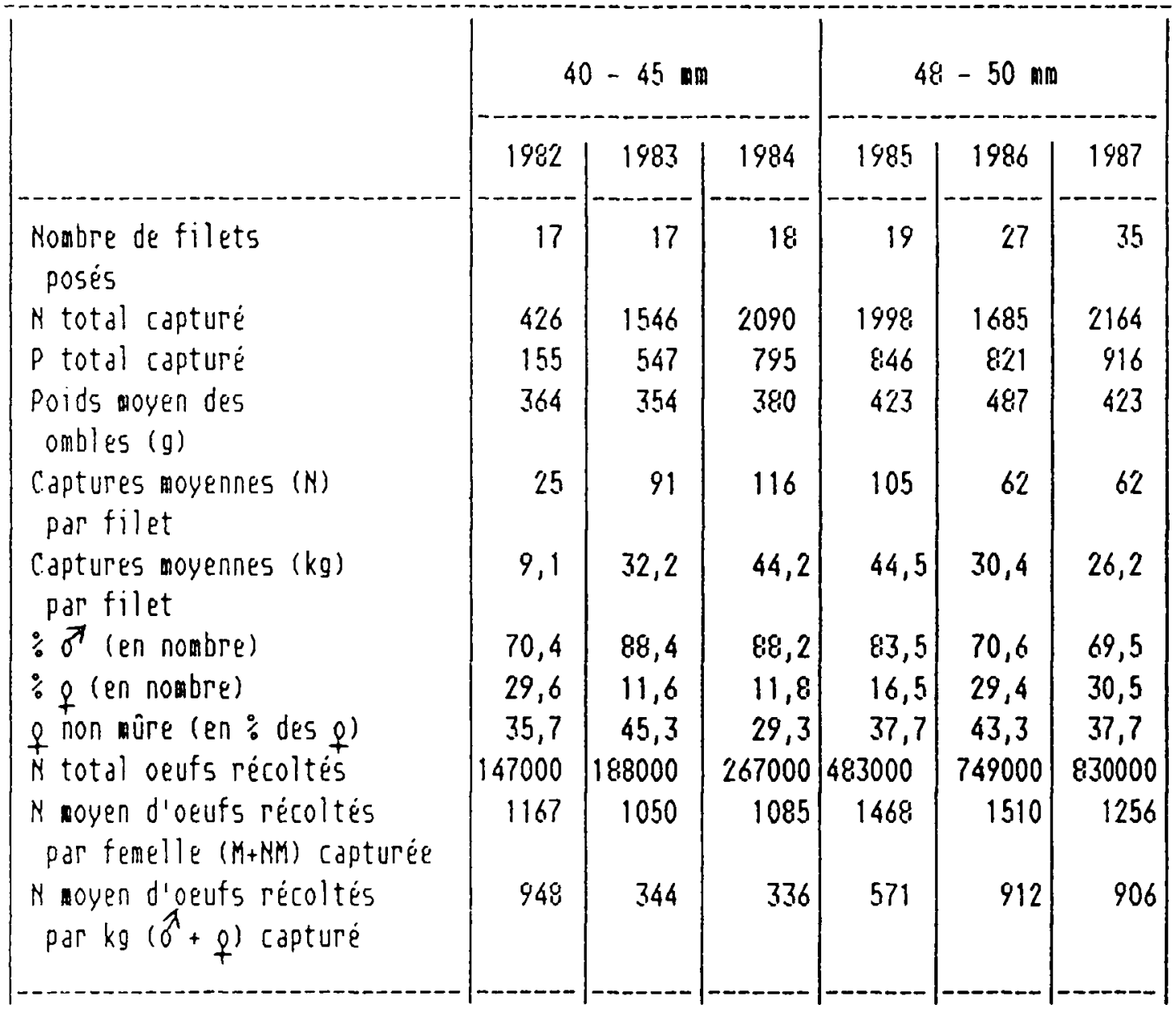

\subsubsection{Récolte d'cuís}

Le nombre total d'œufs récoltés a varié entre 147.000 et 267.000 de 1982 à 1984 . II a ensuite fortement augmenté, passant de 483.000 en 1985 à 830.000 en 1987 (Tab. II). Sur la période 1985-87 le nombre moyen estimé d'œufs récoltés par femelle capturée a été de $1.388 /$ ( 1.256 à 1.510 selon l'année) alors qu'il était de 1.093/ $q$ (1.050 à 1.167 selon l'année) pour la période 1982 à 1984 (Tab. II). Le pourcentage de femelles non müres n'apparaît pas avoir été modifié par le changement du mode de pêche (Tab. II). L'amélioration du rendement des pêches lors des 3 dernières années est en grande partie attribuable à la collecte d'ovules à partir des femelles non müres qui ont été systématiquement stabulées. Ces dernières ont fourni selon l'année de 1985 à 1987 de 19 à $44 \%$ du nombre total d'cufs recueillis à partir des femelles müres. En 1987, la stabulation des femelles non mures a permis de récolter environ $70 \%$ du nombre d'œufs potentiellement récoltables sur ces femelles. 
$N$
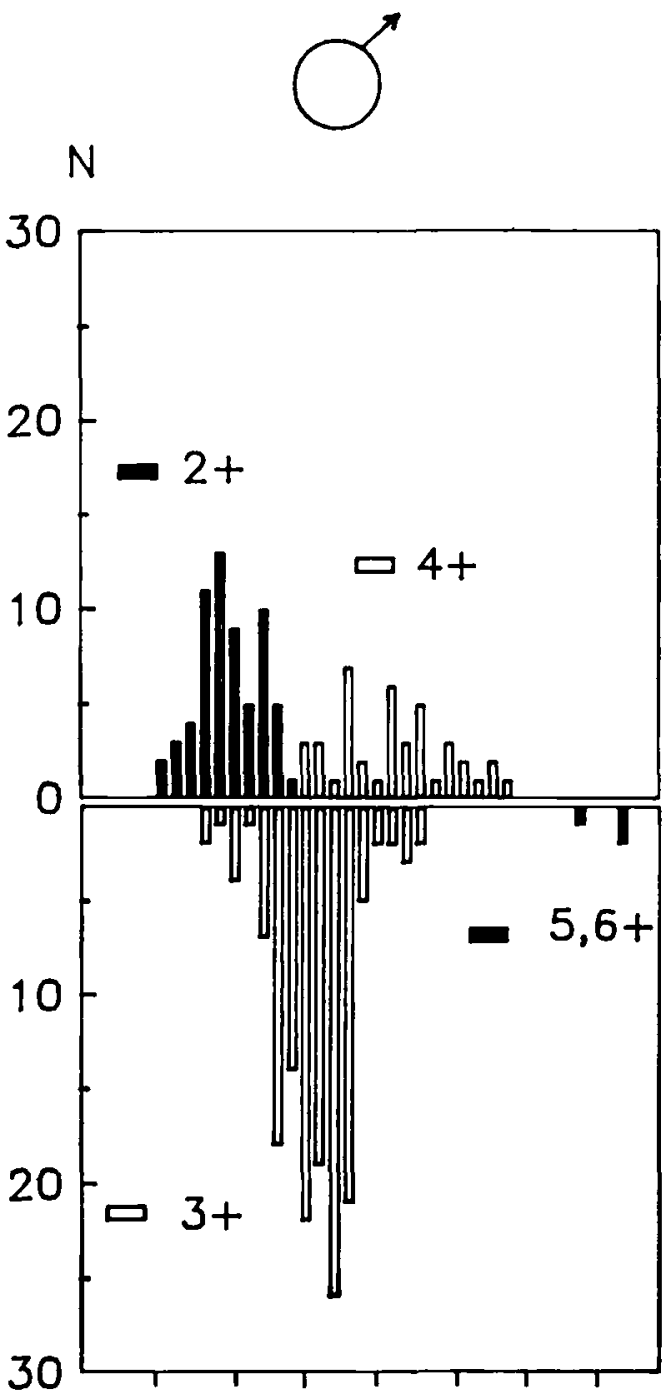

$25303540455055 \mathrm{~cm}$
$N$

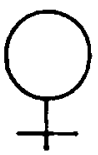

10

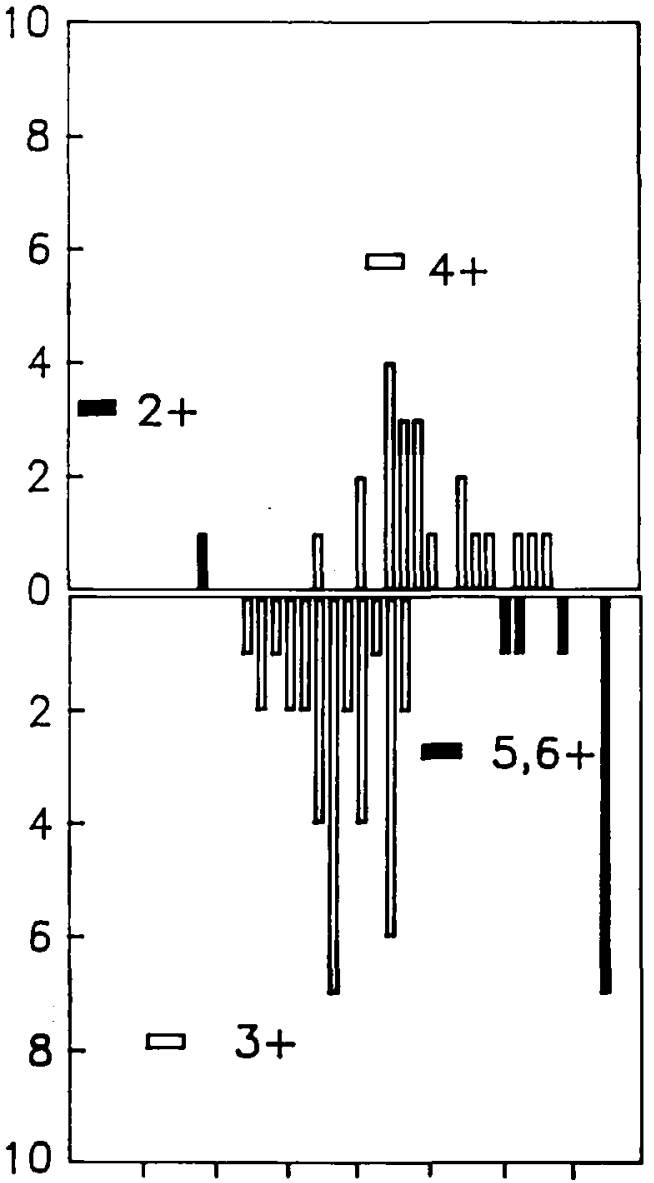

$25303540455055 \mathrm{cM}$

Figure 4 : Structure d'äge $\left(2_{+}, 3_{+}, 4_{+}, \geqslant 5_{+}\right)$et de taille (longueur totale) dans un échantillon de 332 géniteurs d'ombles capturés (maille de $48-50 \mathrm{~mm}$ ) sur les omblières de Meillerie en décembre 1986.

Figure 4 : Structure of age $\left(2+, 3+, 4+, \geqslant 5_{+}\right)$and of size (total lenght) in a sample of 332 charr spawners caught (mesh size $49-50 \mathrm{~mm}$ ) on the spawning grounds of Meillerie in december 1986.

\subsection{Structure d'âge et de taille}

La taille des géniteurs d'ombles capturés a varié respectivement entre 18 et $65 \mathrm{~cm}$ pour les mâles et 26 et $70 \mathrm{~cm}$ pour les femelles. Quels que soient l'année et le maillage des filets on note un décalage entre la structure de taille des mâles et celle des femelles, ces dernières étant en moyenne plus grandes que les mâles capturés (Fig. 3). La structure d'âge et de taille établie pour les géniteurs de 1986 (Fig. 4, Tab. III) montre que ce phénomène est en grande partie lié à la structure d'âge des reproducteurs. En effet, les individus jeunes âgés de 3 ans $(2+)$ représentent près de $27 \%$ des mâles capturés contre seulement $1,5 \%$ des femelles (Tab. III). Les individus $3+(4$ ans) sont les plus 
abondants puisqu'ils représentent, quel que soit le sexe, un peu plus de $50 \%$ des captures. Les individus âgés sont mieux représentés dans les captures de femelles $(31,8 \%$ de $4+$ et $15,2 \%$ de $\geqslant 5+)$ que dans celles des mâles $(15,6$ de 4 + et $1,1 \%$ de $\geqslant 5+)$. La différence dans la structure de taille des mâles et des femelles capturés est renforcée par une différence de croissance (Tab. III). En effet, à âge égal $(3+$ ou $4+$ ) la taille moyenne des femelles capturées $(385 \mathrm{~mm}$ à $3+$ et $448 \mathrm{~mm}$ à $4+)$ est significativement $(p<0,01)$ supérieure à celle des mâles capturés $(359 \mathrm{~mm}$ à $3+$ et $413 \mathrm{~mm}$ à $4+)$. L'examen de la structure de taille de femelles capturées montre que la longueur médiane varie entre 390 et 405 au cours de la période 1985-87 alors qu'elle variait entre 380 et $405 \mathrm{~mm}$ lors des trois années précédentes. La structure de taille des mâles capturés a peu varié entre 1985 et 1987 (Fig. 3). Au cours de ces trois dernières années (maille $48-50 \mathrm{~mm}$ ) la longueur médiane des mâles est voisine de $355 \mathrm{~mm}$ soit supérieure de 25 à $35 \mathrm{~mm}$ selon l'année à celle observée de 1982 à 1984 (Fig. 3), période où le maillage était de $40-45 \mathrm{~mm}$. Lors des essais comparatifs réalisés en 1983 et 1984 avec la pose simultanée de filets de maillage différent, la taille moyenne des mâles capturés (Tab. IV) était significativement ( $p<0,001$ ) plus élevée dans les filets de $48-50 \mathrm{~mm}([=355 \mathrm{~mm})$ que dans ceux de $40-45 \mathrm{~mm}(\bar{L}=329 \mathrm{~mm})$. Lors de ces mêmes essais la taille des femelles capturées n'était pas significativement $(p=0,05)$ différente selon les deux maillages. Par contre, le pourcentage de femelles dans les captures avec les filets de $48-50 \mathrm{~mm}$ était légèrement mais significativement $(p<0,01)$ plus élevé $(16 \%)$ que celui $(11 \%)$ observé dans les filets de $40-45 \mathrm{~mm}$.

Tableaulll : Comparaison de la structure da âge et de la taille moyenne par classe ơáge des mâles et des femelles dans un échantillon de 329 géniteurs d'ombles capturés (maille de $48-50 \mathrm{~mm}$ ) sur les omblières de Meillerie en décembre 1986. Entre parenthèses : intervalle de confiance à $95 \%$.

TableIII : Comparison of age structure and mean total length of males and females in a sample of 329 artic charr spawners caught (mesh size $48-50 \mathrm{~mm}$ ) on the spawning grounds of Meillerie in december 1986. In brackets : confidence limits at $95 \%$ level.

\begin{tabular}{|c|c|c|c|c|c|c|c|c|c|c|}
\hline & \multicolumn{2}{|c|}{$2+$} & \multicolumn{2}{|c|}{$3+$} & \multicolumn{2}{|c|}{$4+$} & \multicolumn{2}{|c|}{$\geq 5+$} & \multicolumn{2}{|c|}{ Total } \\
\hline & $\delta$ & q & $\delta$ & q & $\hat{\sigma}$ & $q$ & $\delta$ & $q$ & $\delta$ & q \\
\hline Nombre & 70 & 1 & 149 & 34 & 41 & 21 & 3 & 10 & 263 & 66 \\
\hline$\because$ du total ou $q$ & 26,6 & 1,5 & 96,7 & 51,5 & 15,6 & 31,8 & 1,1 & 15,2 & 100 & 100 \\
\hline $\begin{array}{l}\text { Longueur poyenne en ma } \\
\text { ( } \pm \text { I.C. d } 95 \%)\end{array}$ & $\begin{array}{l}304 \\
( \pm 6)\end{array}$ & - & $\begin{array}{l}359 \\
( \pm 4)\end{array}$ & $\begin{array}{l}385 \\
( \pm 10)\end{array}$ & $\begin{array}{l}413 \\
( \pm 11)\end{array}$ & $\begin{array}{l}448 \\
( \pm 18)\end{array}$ & - & - & & \\
\hline
\end{tabular}

TableauIV : Bilan des essais comparatifs de captures de géniteurs d'omble en décembre 1983-84 avec deux types de filet $(40-45 \mathrm{~mm}$ et $48-50 \mathrm{~mm})$. (LT) : longueur totale moyenne \pm intervalle de confiance a $95 \%$.

Table IV : Comparative results of catches of charr spawners with two kinds of nets (40-45 mm and $48-50 \mathrm{~mm}$ ) in december 1983-84. (LT) : mean total length \pm confidence limits $95 \%$ level.

\begin{tabular}{|c|c|c|c|c|c|c|c|}
\hline \multirow{2}{*}{$\begin{array}{l}\text { Maille des } \\
\text { filets (m) }\end{array}$} & \multirow{2}{*}{$\begin{array}{l}\text { Nombre } \\
\partial+q\end{array}$} & \multicolumn{3}{|c|}{$q$} & \multicolumn{3}{|c|}{8} \\
\hline & & nowbre & $\because$ & $\operatorname{LT}(\mathrm{mm}) \pm$ I.C. $95 \%$ & nowbre & $\because$ & $\bar{L} T(\mathrm{~m}) \pm$ I.C. 95 \\
\hline $40-45$ & 1241 & 133 & 10,7 & $398 \pm 11$ & 1108 & 89,3 & $329 \pm 3$ \\
\hline $48-50$ & 319 & 52 & 16,1 & $405 \pm 18$ & 267 & 83,7 & $355 \pm 5$ \\
\hline
\end{tabular}



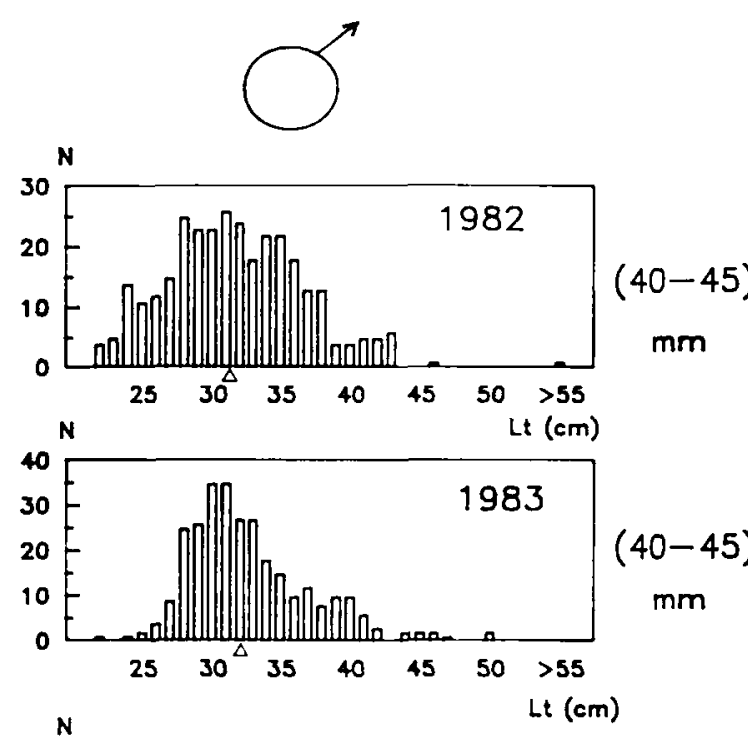

$\mathbf{N}$
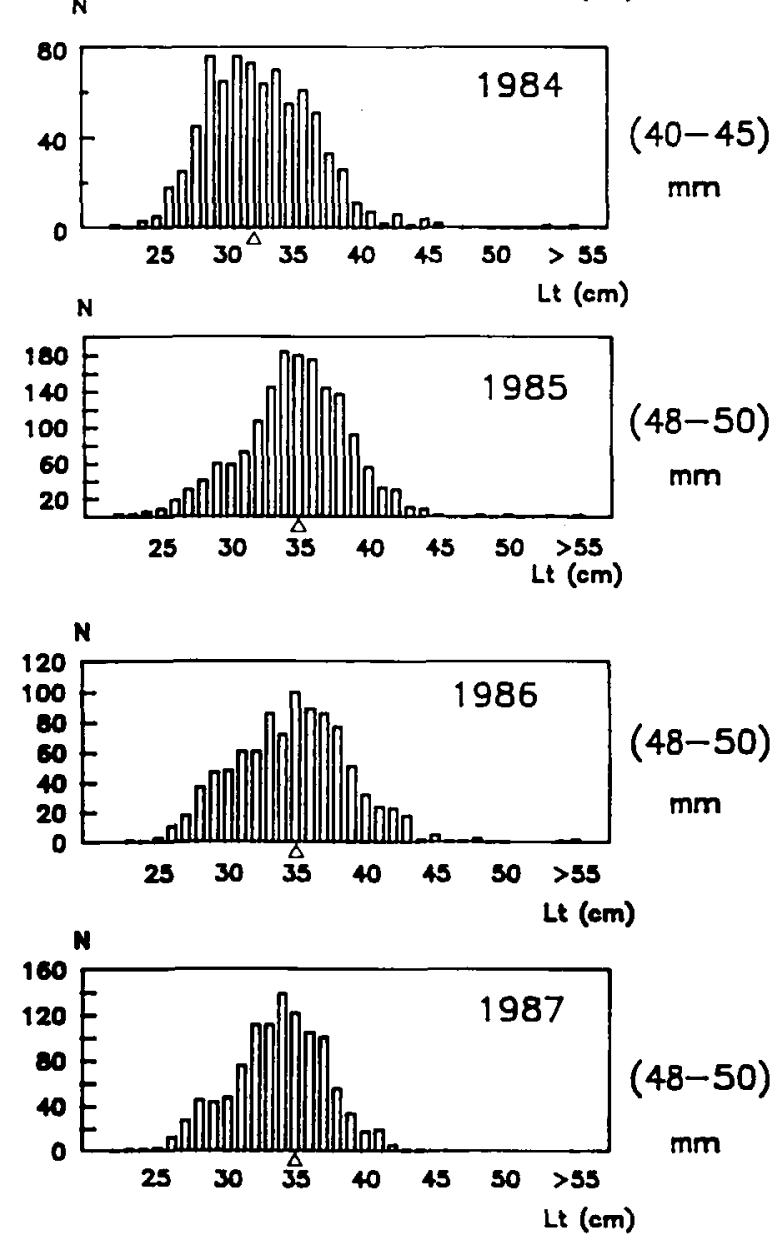
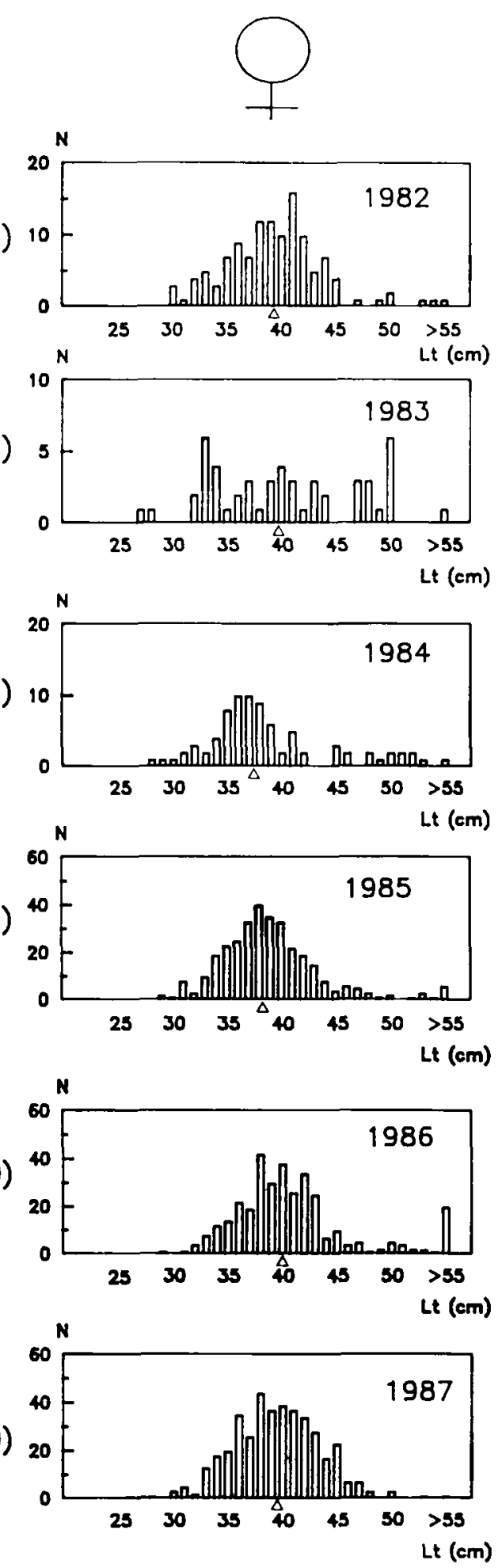

Figure 3 : Structure de taille (longueur totale) en nombre des ombles (sexes séparés) capturés sur les omblières de Melllerle en 1982-83-84 (maille de 40-45 mm), et en 1985-86-87 (maille de $48-50 \mathrm{~mm}$ ). La flèche indlque la longueur médiane pour l'échantillon.

Figure 3 : Size (total length) structure in number for charrs (males or females) caught on the spawning ground of Meillerie in 1982-83-84 (mesh size 40-45 $\mathrm{mm}$ ) and in 1985-86-87 (mesh size of $48-50 \mathrm{~mm}$ ). The arrow indicates the size of the median in the sample. 
3.3. Recaptures d'ombles marqués et contribution du repeuplement dans les captures de géniteurs de la cohorte 1983

\subsubsection{Captures pendant la saison de pêche}

Les quinze premières recaptures contrôlèes d'ombles marqués ont été obtenues dans l'échantillon de 338 ombles (LT: 17 à $43 \mathrm{~cm}$ ) pêchés au filet maillant de la mi-aout à la fin septembre 1985 en baie d'Excenevex (Fig. 1). La contribution des ombles marqués était importante puisqu'ils représentaient $8,7 \%$ des ombles de taille comprise entre 240 et $336 \mathrm{~mm}$ ( $L$ mini et $L$ maxi des ombles marqués) dans l'échantillon examiné. La longueur moyenne des ombles marqués était de $280 \pm 18 \mathrm{~mm}$.

\subsubsection{Captures de géniteurs sur les omblières}

Suivi principal des omblières de Mellierie (Tab. V).

Lors des pêches de reproducteurs (filets $48 \mathrm{~mm}$ ) sur les omblières de Meillerie, les premiers ombles marqués contrôlés sont apparus en décembre 1985, au stade $2+$ (Tab. V). II s'agissait uniquement de mâles; ils avaient une taille moyenne de $321 \pm 18 \mathrm{~mm}$. Des mâles marqués ont continué à être capturés aux stades 3 + et 4 +en décembre 1986 et 87 avec une taille respective de $348 \pm 11$ et de $381 \pm 8 \mathrm{~mm}$. Les premières femelles mûres capturées l'ont été en faible quantité au stade 4 ; elles avaient alors une longueur moyenne de $388 \pm 18 \mathrm{~mm}$.

Lors du suivi des pêches de reproducteurs de 1985 à 1987, la présence d'ombles marqués a été contrôlée sur un nombre total de mâles et de femelles évalué respectivement à 1.087 et 338 individus de la cohorte 1983. Le pourcentage d'ombles marqués n'est pas significativement différent $(p=0,05)$ chez les mâles $(4,05 \%)$ et chez les femelles $(3,85 \%)$.

\section{TableauV : Suivi principal de la présence d’ombles marqués parmi les géniteurs de la cohorte 1983 capturés sur les omblières de Meillerie (France) de 1985 à 1987.}

TableV : Main survey of the occurence of marked charr among spawners of the cohort 1983 caught on the spawning grounds of Meillerie (France) from 1985 to 1987.

\begin{tabular}{|c|c|c|c|c|c|c|}
\hline Sexe & Année & Age & $\begin{array}{c}\text { NT } \\
\text { contrôlé }\end{array}$ & $N$ & $\begin{array}{l}\text { Harqués } \\
\text { : de NT } \\
\pm \text { I.C. à } 95 \%\end{array}$ & $\begin{array}{l}\text { Contribution des } \\
\text { relächers (en : de } \\
\text { NT } \pm \text { I.C. à } 95 \text { : }\end{array}$ \\
\hline$\delta$ & \begin{tabular}{|r}
1985 \\
1986 \\
1987 \\
\\
Total \\
$1985-87$
\end{tabular} & \begin{tabular}{|c|}
$2^{+}$ \\
$3^{*}$ \\
$4^{+}$ \\
\\
Cohorte \\
1983
\end{tabular} & $\begin{array}{r}436 \\
564 \\
87\end{array}$ & $\begin{array}{r}15 \\
22 \\
7\end{array}$ & $\begin{array}{l}3,44 \\
3,90 \\
8,04 \\
4,05 \pm 1,17\end{array}$ & $56,4 \pm 16,3$ \\
\hline q & $\begin{array}{l}1985 \\
1986 \\
1987 \\
\\
\text { Total } \\
1985-87\end{array}$ & $\left|\begin{array}{c}2 * \\
3^{*} \\
4^{*} \\
\text { Cohorte } \\
1983\end{array}\right|$ & $\begin{array}{r}3 \\
228 \\
107\end{array}$ & $\begin{array}{r}0 \\
2 \\
11\end{array}$ & $\begin{array}{c}- \\
0,88 \\
10,28 \\
3,85 \pm 2,05\end{array}$ & $53,6 \pm 28,6$ \\
\hline $8+q$ & $\begin{array}{c}1985 \\
1986 \\
1987 \\
\\
\text { Total } \\
1985-87\end{array}$ & $\mid \begin{array}{c}2^{+} \\
3^{*} \\
4^{+} \\
\text {Cohorte } \\
1983\end{array}$ & $\begin{array}{l}439 \\
792 \\
194\end{array}$ & $\begin{array}{l}15 \\
24 \\
18\end{array}$ & $\begin{array}{l}3,42 \\
3,03 \\
9,28 \\
4,00 \pm 1,02\end{array}$ & $55,7 \pm 14,2$ \\
\hline
\end{tabular}




\section{Suivi complémentaire sur les omblières de Chillon (Tab. VI)}

Le suivi complémentaire réalisé pendant 2 ans sur les omblières suisses de Chillon a mis en évidence la présence de 8 ombles ( $4 \delta$ et $4 q$ ) marqués sur un total de 360 ombles contrôlés. Les effectifs sont trop faibles pour comparer le taux de présence des $\delta$ marqués à celui des femelles marquées. Lorsque l'on regroupe les deux sexes, le pourcentage d’ombles marqués présents sur les omblières de Chillon n'apparaît pas significativement $(p=0,05)$ différent de celui observé sur les omblières de Meillerie que l'on inclue ou non les $2+$ dans la comparaison.

TableauVI : Suivi complémentaire de la présence d’ombles marqués parmi les géniteurs de la cohorte 1983 capturés sur les omblières de Chillon (Suisse) en 1986-87.

TableVI : Complementary survey of the occurence of marked charrs among spawners of the cohort 1983 caught on the spawning grounds of Chillon (Switzerland) in 1986-1987.

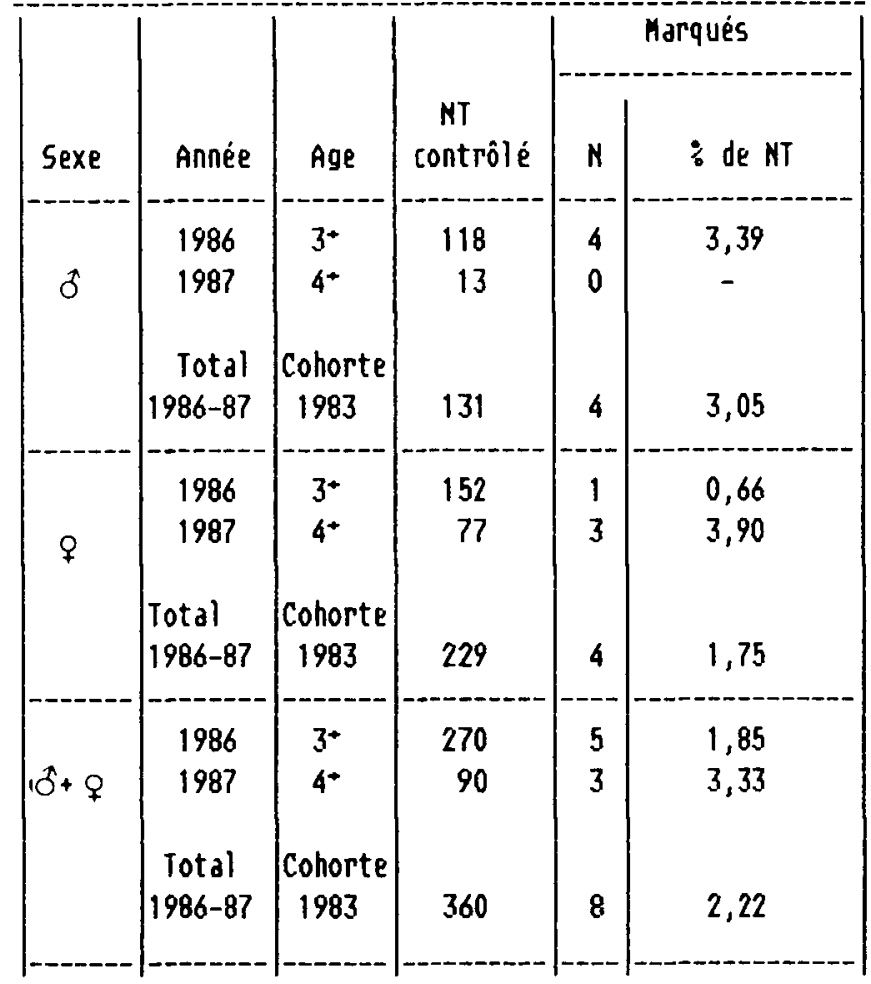

\section{Contribution globale du pacage dans les captures}

de géniteurs de la cohorte 1983 (données regroupées Tab VII)

Le tableau VII précise, pour l'ensemble des contrôles effectués, la contribution des individus issus du lot marqué et leur taille moyenne aux différents âges. Le taux de femelles marquées. présentes en 1987 au stade $4+$ est significativement $(p<0,01)$ plus élevé que celui observé en 1985-86. Par contre, il n'y a pas de différence significative $(p=0,05)$ entre les pourcentages de mâles marqués présents aux différents stades $2+, 3+, 4+$. II n'apparaît pas de différence significative $(p=0,5)$ entre le taux de présence des mâles marqués $(3,9 \%)$ et celui $(3,0 \%)$ des femelles marquées. Si l'on prend en compte l'ensemble des captures de géniteurs contrôlés, le taux de présence d'ombles marqués est de $3.6 \%$. La contribution globale du pacage à la capture de géniteurs de la cohorte 1983 peut être évaluée à $50,7 \pm 12,1 \%$ lorsque l'on fait l'hypothèse d'un comportement identique pour le lot marqué et les lots non marqués.

\section{DISCUSSION}

\subsection{Démarche}

Dans de nombreux lacs en cours d'eutrophisation, le colmatage accru et la diminution de l'oxygénation au niveau des omblières peuvent constituer un facteur limitant la survie des œufs pondus naturellement (RUHLE, 1977 ; CHAMPIGNEULLE, 1985; GILLET, 1985). Une étude en cours (RUBIN, 1987) fait apparaître que trois facteurs de mortalité des œufs sont à considérer sur 
certaines omblières profondes du Léman: la prédation des cufs par la lotte (Lotta lotta), la surdensité en géniteurs sur les omblières de surface restreinte, et surtout le faible taux d'oxygénation.

Tableau VII : Bilan général du suivi de la présence d’ombles marqués parmi les géniteurs de la cohorte 1983 capturés sur les principales omblières connues du Léman de 1985 à 1987.

Table VII : General results of the survey of the occurence of marked charrs among spawners of the cohort 1983 catched on the main spawning grounds of Lake Leman from 1985 to 1987.

\begin{tabular}{|c|c|c|c|c|c|c|c|}
\hline \multirow[b]{2}{*}{ Sexe } & \multirow[b]{2}{*}{ Année } & \multirow[b]{2}{*}{ Age } & \multirow[b]{2}{*}{$\begin{array}{c}\text { NT } \\
\text { contrôlé }\end{array}$} & \multicolumn{3}{|c|}{ Marqués } & \multirow{2}{*}{$\begin{array}{l}\text { Contribution des } \\
\text { relâchers (en : de } \\
\text { NT } \pm \text { I.C. à } 95:\end{array}$} \\
\hline & & & & N & $\begin{array}{c}\text { : de NT } \\
\pm \text { I.C. à } 95:\end{array}$ & $\left\{\begin{array}{l}L T \pm 1 . C . \\
(\text { (w) }) \text { d } 95 \%\end{array}\right.$ & \\
\hline \multirow{5}{*}{$\delta$} & 1985 & $2^{+}$ & 436 & 15 & 3,44 & $321 \pm 18$ & \multirow[b]{5}{*}{$54,9 \pm 15,2$} \\
\hline & 1986 & $3^{+}$ & 682 & 26 & 3,81 & $347 \pm 10$ & \\
\hline & 1987 & $4^{+}$ & 100 & 7 & 7,00 & $381 \pm 8$ & \\
\hline & Total & Cohorte & & & & & \\
\hline & 1985-87 & 1983 & 1218 & 48 & $3,94 \pm 1,09$ & & \\
\hline \multirow{5}{*}{$q$} & 1985 & $2 *$ & 3 & 0 & - & - & \multirow[b]{5}{*}{$41,8 \pm 19,5$} \\
\hline & 1986 & $3+$ & 380 & 3 & 0,79 & - & \\
\hline & 1987 & $4^{+}$ & 184 & 14 & 7,61 & $392 \pm 18$ & \\
\hline & Total & Cohorte & & & & & \\
\hline & $1985-87$ & 1983 & 567 & 17 & $3,00 \pm 1,40$ & & \\
\hline \multirow{5}{*}{$\sigma^{n+}+q$} & 1985 & $2^{+}$ & 439 & 15 & 3,41 & - & \multirow[b]{5}{*}{$50,7 \pm 12,1$} \\
\hline & 1986 & $3^{+}$ & 1062 & 29 & 2,73 & - & \\
\hline & 1987 & $4^{+}$ & 284 & 21 & 7,39 & - & \\
\hline & Total & Cohorte & & & & & \\
\hline & 1985-87 & 1983 & 1785 & 65 & $3,64 \pm 0,87$ & & \\
\hline
\end{tabular}

Pour répondre aux problèmes d'oxygénation réduite en profondeur, certains auteurs ont préconisé des aménagements de frayères (RUHLE, 1977) ou l'introduction de souches d'omble se reproduisant à des profondeurs plus faibles. Il existe en effet selon les souches et les lacs une grande variabilité dans la profondeur de ponte, de l'omble chevalier (JONHSON, 1980 ; GILLET, 1985). Notons que les pêcheurs ont observé qu'actuellement les captures de géniteurs d'ombles sur les omblières françaises du Léman, se font moins profondément qu'il y a 20-30 ans.

Dans le cas du Léman, il a été décidé d'attendre les résultats de l'étude sur les omblières avant de préciser une éventuelle solution quant à leur aménagement. Par contre, le Léman est un cas où la démarche "pacage lacustre" développée par CHAMPIGNEULLE (1985 et 1986) peut être utilisée. II s'agit de fournir un recrutement additionnel en juvéniles lorsque le recrutement naturel, limité, ne permet pas à lui seul de combler le potentiel de production du lac pour l'espèce considérée. Pour éviter tout risque écologique il a été décidé d'utiliser prioritairement les juvéniles produits à partir de géniteurs originaires du Léman. En effet NILSON,(1963) et SVARDSON (1976) signalent de nombreux cas de lacs scandinaves où l'introduction de certaines souches d'omble a conduit à une diminution notable des populations et de la croissance de la truite (Salmo trutta). La démarche de pacage à partir de géniteurs capturés dans le Léman nécessite une optimisation du rendement en œufs des pêches de reproducteurs et une bonne valorisation des cufs prélevés. 


\subsection{Obtention des cufs pour le pacage lacustre}

Au cours de ces dernières annèes, l'abondance de géniteurs sur les omblières de Meillerie a permis l'obtention de plusieurs centaines de milliers d'œufs avec un faible effort de pêche. La forte augmentation des captures par pose notée entre 1982 et 1984 suggère une amélioration récente de la densité en géniteurs. En effet, sur ces mêmes omblières, pêchées avec les mêmes filets de 1979 à 1981 (données DDA non publiées) les captures par pose étaient seulement de 11-12 ombles/pose (4-5 kg/pose) alors que les poses correspondaient alors à la pêche d'un filet pendant 3 à 4 jours consécutifs.

Les pêches de reproducteurs pratiquées il y a 40 ans sur les omblières de Meillerie avaient un rendement en œufs très faible. En effet, selon DUSSART (1952), le rendement était en 1949-50 de seulement 200 à 300 œufs par femelle capturée $(M+N M)$. La pratique de pêche de reproducteurs testée de 1985 à 1987 a permis d'améliorer le rendement en œufs grâce au maintien en vie (1 seule nuit de pose et stabulation) des femelles non mûres capturées. L'existence, dans les captures sur les omblières, d'un sexe-ratio nettement en faveur̃ des mâles, également mis en évidence sur d'autres lacs (RUHLE, 1977 ; RUBIN et BUTTIKER, 1987) est à relier principalement au comportement de fraie de l'omble. En effet, LE CREN et KIPLING (1963) ont montré, grâce à des marquages de géniteurs, que les mâles restent plus longtemps que les femelles sur les omblières. L'influence de la hauteur des filets sur le sexe-ratio des captures reste à étudier dans le cas du Léman. La croissance actuelle de l'omble chevalier au Léman est comparable à celle de la même souche réimplantée sur le lac de Neuchâtel (Suisse) (RUBIN et BUTTIKER, 1987) et légèrement supérieure à ce qu'elle était dans le Léman il y a 50 ans (DUSSART, 1952).

Une autre voie possible, permettant la fourniture d'œufs pour le pacage lacustre est la constitution d'un stock de géniteurs en pisciculture en partant d'œufs issus des pêches de reproducteurs. Les adultes peuvent être obtenus avec les techniques classiques de la salmoniculture en eau froide (LAURENT, 1982a; CHAMPIGNEULLE, 1986). Cependant, jusqu'à une période récente, l'obtention de pontes de qualité bonne et régulière posait problème pour les géniteurs produits en pisciculture (PAPST et HOPKY, 1984). Des travaux récents menés sur l'omble du Léman (Institut de Limnologie, 1986; GILLET, données non publiées) ont permis de maîtriser l'aspect qualité des pontes. Il devrait donc être possible d'accroitre les possibilités de fourniture d'œufs issus de géniteurs produits en pisciculture. Cet apport viendrait en complément des œufs issus des pêches contrôlées de reproducteurs, ces dernières étant utiles à la bonne gestion de la population d'ombles: suivi de l'état du stock, contrôle de l'efficacité du pacage (marquages), exploitation contrôlée (quotas)

\subsection{Efficacité du pacage lacustre}

II existe très peu de données publièes sur l'efficacité des relâchers (au stade d'estivaux en particulier) d'ombles dans les grands lacs (Tab. VIII). Plusieurs auteurs (RUNNSTROM, 1951; GRIMAS et al., 1972) signalent la très faible efficacité des relâchers d'alevins vésiculés alors que les taux de recaptures augmentent lorsque les juvéniles sont prégrossis en pisciculture (Tab. VIII).

D'après la présente étude, il apparaît qu'au minimum $50 \%$ des géniteurs d'ombles de la cohorte 1983 capturés sur les omblières du Haut-léman proviennent des relâchers d'estivaux. Les contrôles du marquage et l'établissement des structures d'âge ont été réalisés sur les ombles sexés puisque en accord avec DUSSART (1952), RUHLE (1977), RUBIN et BUTTIKER (1987) la taille des femelles capturées est légèrement supérieure à celle des mâles. Les femelles maturent plus tardivement que les mâles en accord avec ce qui est observé en pisciculture (GILLET, comm. person.) ou dans d'autres lacs (RUBIN et BUTTIKER, 1987). Pour limiter au maximum les biais liés à ce dernier facteur et à la technique d'échantillonnage au filet, le bilan a été réalisé après 3 ans de suivi depuis le stade 2 + d'entrée dans la pêcherie jusqu'au stade $4+$. Il n'a pas été poursuivi au-delà car le nombre contrôlable devient trop faible et les écailles deviennent très difficile à lire.

Le lot marqué a été élevé et déversé à une grande distance des omblières afin de limiter les biais possibles liés à une stabilisation à proximité de ces dernières ainsi que d'éventuelles conditions favorables à la mise en place d'un processus de "homing". Ce dernier phénomène reste encore mal connu dans le cas des souches d'ombles se reproduisant en lac. FROST (1963), LE CREN et KIPLING (1963) indiquent l'attachement des géniteurs d'omble à la même omblière lors de fraies multiples. Cependant, mis à part le cas des géniteurs se reproduisant en rivière (FROST, 1963), il n'y a pas de données publiées sur le homing d'ombles (issu de la reproduction naturelle) au sens du retour des géniteurs aux omblières lacustres fréquentées au stade juvénile (œufs, début de nourrissage). La présente étude et des nouveaux suivis (RUBIN, CHAMPIGNEULLE, données non publiées) montrent que les juvéniles déversés au stade d'estivaux peuvent venir sur des omblières qui ne sont pas les omblières parentales. D'après une étude en cours (RUBIN, comm. person.) il apparaît que le retour préférentiel à une zone d'omblière donnée est favorisé lorsque le déversement a été réalisé à proximité de cette dernière. 
Tableau VIII: Quelques donnees bibliographiques sur l'impact (recaptures) des relâchers d'omble chevalier dans quelques grands lacs europeens.

Table VIII : Some published data about the efficiency (recaptures) of restocking with artic charrs in some great european lakes.

\begin{tabular}{|c|c|c|c|c|c|}
\hline \multirow[b]{2}{*}{ AUTEURS } & \multirow{2}{*}{$\begin{array}{c}\text { LAC } \\
\text { (Surface) } \\
\text { ha }\end{array}$} & \multicolumn{2}{|c|}{ RELACHERS } & \multicolumn{2}{|c|}{ RECAPTURES } \\
\hline & & $\left|\begin{array}{c}\text { Stade } \\
\text { Tailler(u) }\end{array}\right|$ & $\begin{array}{l}\text { Densités } \\
\text { (n/ha) }\end{array}$ & $\begin{array}{c}\text { node } \\
\text { appréciation }\end{array}$ & $\begin{array}{l}\text { Niveau des } \\
\text { recaptures }\end{array}$ \\
\hline $\begin{array}{l}\text { GRIMAS et d. } \\
1972\end{array}$ & $\begin{array}{l}\text { Uättern } \\
191.200\end{array}$ & $\begin{array}{l}\text { devins } \\
\text { vésiculés }\end{array}$ & $8-16$ & $\begin{array}{l}\text { Evolution des lächers } \\
\text { et des captures }\end{array}$ & Négl igeable \\
\hline $\begin{array}{l}\text { RUHASTROM } \\
\text { 1959 }\end{array}$ & $\begin{array}{l}\text { Torrön } \\
9.500\end{array}$ & $\begin{array}{l}\text { alevins } \\
\text { vésiculés }\end{array}$ & $32-74$ & $\begin{array}{l}\text { Evolution des lâchers } \\
\text { et des captures }\end{array}$ & $\begin{array}{l}\text { pas d'effet } \\
\text { significatif }\end{array}$ \\
\hline $\begin{array}{l}\text { HARTMANN } \\
1986\end{array}$ & $\begin{array}{l}\text { Constance } \\
53.900\end{array}$ & $\begin{array}{l}\text { alevins } \\
\text { nourris }\end{array}$ & & $\begin{array}{l}\text { Evolution parallèle } \\
\text { relâchers-captures }\end{array}$ & Effect suspecté \\
\hline $\begin{array}{l}\text { PEDROLI } \\
1983\end{array}$ & $\begin{array}{c}\text { Neuchàtel } \\
21.500\end{array}$ & $\begin{array}{l}\text { alevins } \\
\text { nourris } \\
(7-9) \\
\text { origine: } \\
\text { Léan }\end{array}$ & 1,4 & $\begin{array}{l}\text { Apparition de captures } \\
\text { après une péride sans } \\
\text { captures. Pas de } \\
\text { narquage }\end{array}$ & $\begin{array}{l}\text { Très él evé } \\
23:\end{array}$ \\
\hline $\begin{array}{l}\text { CRIMAS et al. } \\
1970\end{array}$ & $\begin{array}{l}\text { Uättern } \\
191.200\end{array}$ & $1-(15)$ & - & Harquage & Peu de recaptures \\
\hline $\begin{array}{l}\text { GRIMAS et al. } \\
1972\end{array}$ & $\begin{array}{l}\text { Uättern } \\
191.200\end{array}$ & $\begin{array}{l}\geq 2 \\
(20-25)\end{array}$ & - & Marquage & $\begin{array}{l}\text { hoy. } 20 \%(11-24 \%) \\
8 \text { à } 33 \mathrm{~kg} / 1000 \\
\text { oubles rel àchés }\end{array}$ \\
\hline $\begin{array}{l}\text { LAURENT } \\
1982 \mathrm{~b}\end{array}$ & $\begin{array}{l}\text { Léman } \\
58.240\end{array}$ & $1+(13)$ & - & Harquage & $13:$ \\
\hline Présente étude & $\begin{array}{l}\text { Lèuan } \\
58.240\end{array}$ & $0 \cdot(3-10)$ & 6,3 & $\begin{array}{l}\text { Marquage }(3-5 \mathrm{ca}) \text { et } \\
\text { échantillonnage des } \\
\text { captures }\end{array}$ & $\begin{array}{l}\text { Prewiere estiad- } \\
\text { tion : niniaun } \\
\text { voisin de 20kg/ } \\
1000 \text { estivaux }\end{array}$ \\
\hline
\end{tabular}

Le tableau VI précise un certain nombre de facteurs, allant tous dans le même sens, et quiont pu conduire à une sous-évaluation de la contribution du repeuplement. Une importante étude en cours (RUBIN, comm. person.) portant sur les cohortes 1984 et 1985 confirme cette hypothèse. La présente étude ayant porté sur les ombles présents sur les principales omblières du Léman permet d'avancer l'hypothèse qu'au minimum $50 \%$ de la cohorte 1983 provient du pacage lacustre. Sous cette hypothèse et dans le contexte actuel du Léman (15 tonnes de captures totales/an : amateurs professionnels, 300 à 370.000 estivaux lâchés annuellement de 1983 à 1985), il est possible d'avancer que la valeur de vente des ombles capturés issus du pacage lacustre dépasse les coüts de production des juvéniles. Le niveau minimum des recaptures se situerait à environ $20 \mathrm{~kg}$ pour 1.000 alevins nourris relâchés. L'augmentation récente du nombre d'estivaux relâchés (niveau 500-700.000/an de 1986 à 88 ) devrait se traduire par une augmentation des captures dans les années à venir sous réserve que l'effort de pêche soit maintenu et que la mortalité naturelle n'augmente pas fortement. 


\section{CONCLUSION}

La présente étude a montré que l'omble chevalier est une espèce potentiellement intéressante pour le pacage lacustre dans le Léman. Un important effort de recherche reste cependant nécessaire pour optimiser la production de juvéniles, les caractéristiques des relâchers (origine, site, densité, période, taille...) et l'exploitation des adultes produits. la technique de marquage magnétique, praticable dès la taille de $25-35 \mathrm{~mm}$ (CHAMPIGNEULLE, 1987) devrait permettre de tels travaux. Dans un lac comme le Léman, une telle recherche implique un programme de grande envergure afin de prendre en compte les fluctuations interannuelles, la taille du lac (lots importants à marquer) et les biais possibles liés à des hétérogénéités dans les recaptures en fonction du mode de pêche (filet, traine) et des caractéristiques des relâchers. L'étude de la reproduction naturelle devra être poursuivie en vue notamment de "hiérarchiser" l'impact de divers facteurs sur le succès de la fraie naturelle (déficit en oxygène, prédation, surdensité sur les omblières existantes) et donc de proposer des opérations complémentaires d'aménagement ou de gestion.

\section{REMERCIEMENTS}

L'étude a ètè rendue possible grâce à une collaboration ètroite entre les pêcheurs, l'ensemble des techniciens du Service Pêche de la DDA de Haute Savoie à Thonon, les gardes du Conseil Supérieur de la Pêche pour le Léman, le Service de la Conservation de la Faune en Suisse (M. J.F. RUBIN) et l'ensemble de l'équipe ichtyologie de I'INRA à Thonon.

\section{BIBLIOGRAPHIE}

CHAMPIGNEULLE A., GERDEAUX D. et GILLET C., 1983. Les pêches exceptionnelles d'ombles et de corégones dans les eaux françaises du Léman en 1982. Rapp. Inst. Limnol., 6, 15 p.

CHAMPIGNEULLE A. et ESCOMEL J., 1984. Note technique. Marquage de Salmonidés de petite taille par ablation de l'adipeuse ou des nageoires pelviennes. Bull. Fr. Piscic., 293-294, 52-58.

CHAMPIGNEULLE A., 1985. Analyse bibliographique des problèmes de repeuplement en omble chevalier (Salvelinus alpinus), truite fario (Salmo trutta) et corégones (Coregonus sp.) dans les grands plans d'eau 187-217 in D. GERDEAUX et R. BILLARD Ed., Gestion piscicole des lacs et retenues artificielles. INRA, Paris.

CHAMPIGNEULLE A., 1986. Connaissance et maîtrise des repeuplements de Salmonidés en lacs. Restauration de stocks et pacage lacustre. Rapp. Inst. Limnol. Thonon, 24, 23 p.

CHAMPIGNEULLE A., 1987. Note technique. Marquage d'ombles chevaliers (Salvelinus alpinus L.) de petite taille par injection de micromarques magnétisées. Bull. Fr. Pêche Piscic.,304. 22-31.

CIPEL, 1984. Le Léman. Synthèse des travaux de la CIPEL de 1957 à 1982. Lausanne, 1 vol., $650 \mathrm{p}$.

CRETTIEZ M.J., 1906. La culture de l'omble chevalier du lac Léman. Métis et hybrides de ce salmonide. C.R. Assoc. Fr. Av. Sc., Congrès de Lyon, sect. Zool., 498-506.

DUSSART B., 1952. L'omble chevalier du Léman (Salvelinus alpinus L.) Ann. Stat. Cent. Hydrobiol. Appl., 4, 353-377.

FROST W.F., 1963. The homing of charr (Salvelinus willughbii G.) In Windermere. Animal Behaviour, 11, 74-82.

GILLET C., 1985. Le déroulement de la fraie des principaux poissons lacustres, 167-175, in D. GERDEAUX et R. BILLARD Ed., Gestion piscicole des lacs et retenues artificielles, INRA, Paris.

GRIMAS U., NILSSON N.A. and WENDT C., 1972. Lake Vättern : effect of exploitation, eutrophication and introductions on the salmonid community. J. Fish. Res. Board Can., 29, 807-817.

HARTMANN J., 1986. Besatzerfolg ? Der Fishwirt, 36, 33-35

INSTITUT DE LIMNOLOGIE, 1986. ATP INRA No 4355. Connaissance et gestion des écosystèmes lacustres subalpins. Rapport d'activité 1983-85. Travaux en ichtyologie. Thonon, 1 vol., 59 p.

JOHNSON L., 1980. The artic charr (Salvelinus alpinus L.) In BALON E.K. Ed., Charrs, Salmonids fishes of the genus Salvelinus, 15-98. Junk Publ., La Haye.

LAURENT P.J., 1972. Lake Leman : effects of exploitation, eutrophication, and introductions on the salmonid community. J. Fish. Res. Bd Canada, 29, 867-875.

LAURENT P.J., 1982a. Quelques données sur l'élevage de l'omble chevalier (Salvelinus alpinus L.). Bull. Liaison CSP, 21, 10-13.

LAURENT P.J., 1982b. Résultats de déversements dans le Léman d'ombles marqués. Bull. Fr. Piscic., 285, 210-220. 
LE CREN E.D. and KIPLING C., 1963. Some marking experiments on spawning populations of Char. Spec. Publ ICNAF, 4, 130-139.

NILSON N.A., 1963. Interaction between trout and charr. Trans. Am. Fish. Soc., 92, 276-285.

PAPST M.H. and HOPKY G.E., 1984. Development of an artic charr (Salvelinus alpinus L. ) brood stock. Aquaculture, 43, 323-331.

PEDROLI J.C., 1983. La réintroduction de l'omble chevalier (Salvelinus alpinus) dans le lac de Neuchâtel. Bull. Fr. Piscic., 290, 158-160.

RUBIN J.F., 1987. Étude des omblières du Léman avec l'aide du sous-marin "F.A. FOREL". Rapport interne Conservation de la Faune, Lausanne, 1 vol., 12 p.

RUBIN J.F. et BUTTIKER B., 1987. Croissance et reproduction de l'omble chevalier (Salvelinus alpinus L.) dans le lac de Neuchâtel (Suisse). Schweiz. Z. Hydrol., 49, 51-61.

RUHLE C., 1977. Biologie und Bewirtschaftung des Seesaiblings (Salvelinus alpinus L.) im Zugersea. Schweiz. Z. Hydrol., 39, 12-45.

RUNNSTROM S., 1951. The population of charr (Salvelinus Alpinus) in a regulate lake. Rep. Inst. Freshwater Res. Drottningholm, 32, 66-78.

SVARDSON G., 1976. Interspecific population dominance in fish communities of Scandinavian lakes. Rep. Inst. Freshwater Res. Drottningholm, 55, 144-171. 